\title{
Adverse Events from Emollient Use in Eczema: A Restricted Review of Published Data
}

\author{
Alisha Bhanot · Alyson Huntley $\cdot$ Matthew J. Ridd (D
}

Received: December 20, 2018 / Published online: February 15, 2019

(C) The Author(s) 2019

\begin{abstract}
Atopic dermatitis/eczema is a chronic inflammatory skin condition, and emollients are the first-line treatment. Despite their widespread use, there is uncertainty about the frequency and type of adverse events associated with different emollients. We conducted a restricted review of published data on adverse events associated with emollient use in eczema. Medline (Ovid) was searched from inception (1946) to June 2018. All types of studies, with the exception of reviews, were included. Eligibility was assessed using a two-stage screening process against inclusion and exclusion criteria. References of all included papers were screened for any additional eligible papers. Data were subsequently extracted from all eligible publications. A limited body of data were found in the published data: 24 papers reported on adverse events with 29 different emollients (3
\end{abstract}

Enhanced Digital Features To view enhanced digital features for this article go to https://doi.org/10.6084/ m9.figshare.7637108.

Electronic supplementary material The online version of this article (https://doi.org/10.1007/s13555019-0284-3) contains supplementary material, which is available to authorized users.

A. Bhanot · A. Huntley · M. J. Ridd ( $\square)$

Population Health Sciences, University of Bristol, Bristol, UK

e-mail: m.ridd@bristol.ac.uk containing urea, 5 containing ceramide, 4 containing glycerol, 4 were herbal and 13 contained "other" ingredients). Interpretation of the results and comparison of the emollients were difficult due to poor reporting and missing data. Many publications contained no data at all on adverse events, and no study reported serious treatment-related adverse events for any emollient. The proportion of participants in the studies experiencing treatment-related adverse events varied between 2 and 59\%. The most common adverse events were skin related and often mild. The range of participants experiencing non-treatment-related adverse events varied between 4 and 43\%. From this restricted review, clinicians and patients can be reassured that the emollients studied appear to be generally safe to use. Better studies and reporting of adverse events associated with emollients in common use are needed.

Keywords: Adverse events; Emollients; Atopic dermatitis; Atopic eczema; Moisturizers

\section{INTRODUCTION}

Atopic eczema (atopic dermatitis, hereafter "eczema") is a chronic inflammatory skin condition characterized by red, itchy skin lesions [1]. There is a stepwise approach to the treatment of eczema, depending on disease severity 
and response [2]. Emollients are topical therapies which act to "soothe, smooth and hydrate the skin" [3]. They are the first-line treatment for eczema across all severities and should be used continually with and in larger quantities than other topical treatments [2].

Given the widespread and long-term use of emollients in treating eczema, an understanding of just how common adverse events are associated with emollients is important. However, the reporting of adverse events associated with topical eczema therapies has focused on the use of corticosteroids and calcineurin inhibitors, such as tacrolimus [4, 5]. A recent Cochrane review of emollients used to treat eczema only included adverse event data from randomized control trials (RCTs) [1].

The aim of this study was to conduct a restricted review [6] into adverse events associated with the use of emollients in eczema.

\section{METHODS}

\section{Literature Search}

The Medline database (Ovid) was searched from inception (1946) to June 2018 for adverse events associated with emollient use in patients with eczema. A search strategy was developed using keywords from the electronic database, which combined search terms for eczema, emollients and adverse events (see Electronic Supplementary Material [ESM] Appendix 1). Duplicate publications were removed, and one of the authors (AB) assessed eligibility by screening titles and abstracts against the inclusion and exclusion criteria. All identified papers were then obtained and read in full. References of all included papers were screened for any additional eligible papers. When $\mathrm{AB}$ was uncertain about the inclusion or exclusion of a paper, its eligibility was discussed with a second author (MR) and agreement was reached. The reasons for exclusion were recorded.

\section{Eligibility Criteria}

Studies investigating "leave-on" emollients for the treatment of participants with atopic eczema/dermatitis of any age were included in this restricted review and included RCTs, cohort studies, case-control studies and case reports/ series. Studies excluded were: those not in humans; those not published in English; those in which the emollient was not used for the treatment of eczema or was mixed with other topical therapies for eczema (e.g. topical corticosteroids); and those in which adverse events related to emollient use were not included in the title or abstract.

\section{Data Extraction}

One author $(\mathrm{AB})$ developed and piloted a data extraction tool before applying it to all the included papers. The main outcome measure of interest was the frequency and nature of adverse events associated with emollient use. Expected adverse events related to emollient use included pruritus, rash, erythema, pain, burning and hypersensitivity. Type and name of emollient, study type, setting, patient population and disease diagnostic criteria (if any) applied were also recorded.

\section{Compliance with Ethics Guidelines}

This article is based on previously conducted studies and does not contain any studies with human participants or animals performed by any of the authors, so ethical approval was not required.

\section{RESULTS}

\section{Database Search}

A total of 897 papers were initially identified; following the removal of duplicate studies, 892 titles and abstracts were screened for eligibility. The full text of 102 papers were then screened, and 80 papers excluded. Two papers were added from the references of the included papers 


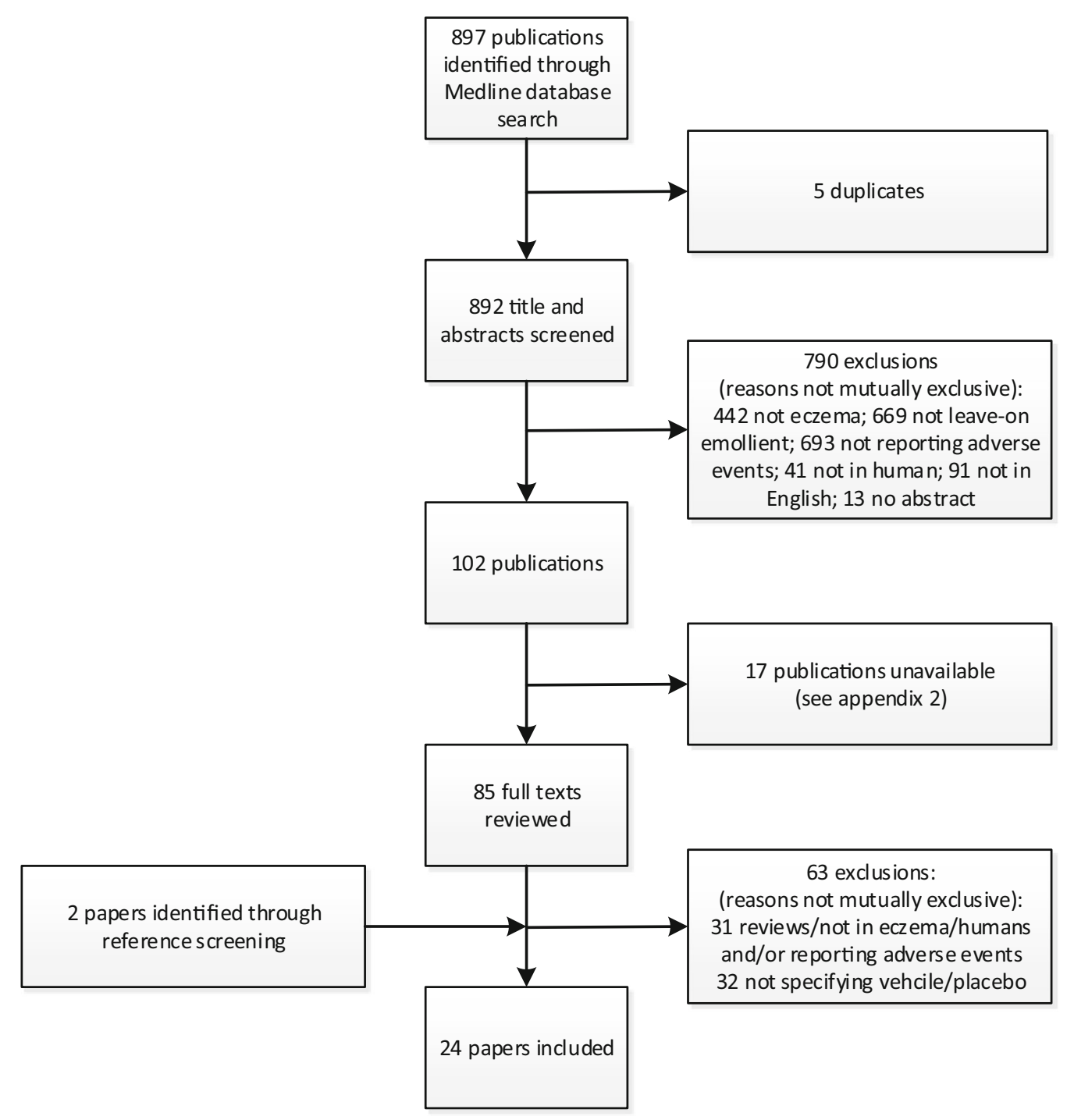

Fig. 1 Flowchart of study selection

which resulted in a total of 24 papers included in the review (see Fig. 1).

\section{Study Characteristics}

The characteristics of the included papers are summarized in Table 1 . Most studies were RCTs $(17,71 \%)$ [7-23], followed by non-randomized interventional studies $(5,21 \%)$ [24-28]. There was one cohort [29] and one case-control study [30]. Most of the papers $(13,54 \%)$ did not specify the settings in which the studies were conducted
$[7,11,13,14,18-20,22,23,25-27]$; however, ten studies took place in a specialist setting (7 secondary care centers $[9,10,12,15,24,28,30]$, 2 research centers $[8,21]$ and 1 mobile dermatological center [16]). One study took place in both a secondary care and a research centre [17]. No studies took place in a primary care/community setting (see Table 1 ).

There were ten $(42 \%)$ studies involving just children [10-12, 14, 15, 17, 18, 21, 27, 28], five (21\%) with only adults $[7,8,19,22,23]$ and nine (38\%) involving both children and adults $[9,13,16,20,24-26,29,30]$. Eleven (46\%) 
Table 1 Characteristics of the papers included in the review

\begin{tabular}{|c|c|c|c|c|c|}
\hline References & Emollient(s) & $\begin{array}{l}\text { Study setting } \\
\text { (country) }\end{array}$ & $\begin{array}{l}\text { Non-emollient } \\
\text { comparator }\end{array}$ & $\begin{array}{l}\text { Patient } \\
\text { population }\end{array}$ & $\begin{array}{l}\text { Eczema diagnostic } \\
\text { criteria }\end{array}$ \\
\hline $\begin{array}{l}\text { Chamlin et al. } \\
\text { [28] }\end{array}$ & TriCeram Cream & $\begin{array}{l}\text { Pediatric dermatology } \\
\text { clinics (USA) }\end{array}$ & - & $\begin{array}{l}\text { Stubborn-to- } \\
\text { recalcitrant } \\
\text { AD } \\
\text { Aged } 1-12 \text { years } \\
n=24\end{array}$ & Unspecified \\
\hline $\begin{array}{l}\text { Chishti et al. } \\
\text { [30] }\end{array}$ & Dermovix & $\begin{array}{l}\text { ShifaulMulk } \\
\text { Memorial-Hospital } \\
\text { and Al-Rasheed } \\
\text { Darushifa Malkani } \\
\text { Liaqut Pur } \\
\text { (Pakistan) }\end{array}$ & $\begin{array}{l}\text { Betnovate } \\
\text { N-Cream }\end{array}$ & $\begin{array}{l}\text { AD } \\
n=60 \\
\text { Aged } 2 \text { years } \\
\quad \text { to }>40 \text { years }\end{array}$ & Unspecified \\
\hline Draelos [27] & $\begin{array}{l}\text { Atrapro } \\
\text { Antipruritic } \\
\text { HydroGel }\end{array}$ & Unspecified & - & $\begin{array}{l}\text { Mild-moderate } \\
\quad \text { AD } \\
\text { Aged } \\
18-65 \text { years } \\
n=17\end{array}$ & Hanifin and Rajka \\
\hline $\begin{array}{l}\text { Draelos and } \\
\text { Raymond } \\
{[25]}\end{array}$ & NeoCera & Unspecified & - & $\begin{array}{l}\text { Mild-to- } \\
\text { moderate } \\
\text { atopic } \\
\text { dermatitis or } \\
\text { other xerotic/ } \\
\text { pruritic } \\
\text { dermatoses } \\
\text { Aged } 1-86 \text { years } \\
n=50\end{array}$ & Unspecified \\
\hline $\begin{array}{l}\text { Boguniewicz } \\
\text { et al. [21] }\end{array}$ & Atopiclair Cream & 7 Study centers (US) & $\begin{array}{l}\text { "Vehicle" } \\
\quad \text { (unspecified) }\end{array}$ & $\begin{array}{l}\text { AD } \\
\text { Aged } 6 \text { months } \\
\text { to } 12 \text { years } \\
n=142\end{array}$ & Hanifin and Rajka \\
\hline $\begin{array}{l}\text { Boralevi et al. } \\
\text { [11] }\end{array}$ & $\begin{array}{l}\text { Dexeryl (glycerol } \\
\text { containing } \\
\text { emollient) }\end{array}$ & Unspecified & "Vehicle" & $\begin{array}{l}\text { AD } \\
\text { Aged } 2-6 \text { years } \\
n=251\end{array}$ & UK Working Party \\
\hline
\end{tabular}


Table 1 continued

\begin{tabular}{|c|c|c|c|c|c|}
\hline References & Emollient(s) & $\begin{array}{l}\text { Study setting } \\
\text { (country) }\end{array}$ & $\begin{array}{l}\text { Non-emollient } \\
\text { comparator }\end{array}$ & $\begin{array}{l}\text { Patient } \\
\text { population }\end{array}$ & $\begin{array}{l}\text { Eczema diagnostic } \\
\text { criteria }\end{array}$ \\
\hline $\begin{array}{c}\text { Bissonnette } \\
\text { et al. [7] }\end{array}$ & $\begin{array}{l}\text { Iso-Urea (5\% urea } \\
\text { moisturizer) } \\
10 \% \text { urea lotion }\end{array}$ & Unspecified & - & $\begin{array}{l}\text { Mild-moderate } \\
\quad \text { AD } \\
\text { Aged } \\
18-70 \text { years } \\
n=100\end{array}$ & Unspecified \\
\hline $\begin{array}{l}\text { Gandy et al. } \\
\text { [13] }\end{array}$ & $\begin{array}{l}\text { CHD-FA 3.5\% } \\
\text { (carbohydrate- } \\
\text { derived fulvic } \\
\text { acid) } \\
\text { "Placebo } \\
\text { emollient" } \\
\text { (unspecified) }\end{array}$ & Unspecified & - & $\begin{array}{l}\text { Eczema } \\
>2 \text { years } \\
n=36\end{array}$ & Unspecified \\
\hline Haider [10] & $\begin{array}{l}\text { 10\% Sodium } \\
\text { cromoglycate } \\
\text { ointment } \\
\text { Placebo ointment } \\
\text { (white soft } \\
\text { paraffin) }\end{array}$ & $\begin{array}{l}\text { Bury General } \\
\text { Hospital (England) }\end{array}$ & - & $\begin{array}{l}\text { Chronic atopic } \\
\text { eczema } \\
\text { Children } \\
n=42\end{array}$ & Unspecified \\
\hline $\begin{array}{l}\text { Hashizume } \\
\text { et al. [23] }\end{array}$ & $\begin{array}{l}N \text {-acetyl- } \\
\text { hydroxyproline } \\
\text { cream (AHYP; } \\
\text { contains glyerin } \\
\text { and glyceryl } \\
\text { stearate) }\end{array}$ & Unspecified & $\begin{array}{l}\text { "Control cream” } \\
\text { (unspecified) }\end{array}$ & $\begin{array}{l}\text { Slight AD } \\
\text { Aged } \\
20-49 \text { years } \\
n=14\end{array}$ & $\begin{array}{l}\text { Atopic dermatitis } \\
\text { treatment } \\
\text { guidelines of the } \\
\text { Japanese } \\
\text { Dermatological } \\
\text { Association }\end{array}$ \\
\hline $\begin{array}{l}\text { Hlela et al. } \\
\text { [12] }\end{array}$ & $\begin{array}{l}\text { Cetomacrogrol } \\
\text { Emulsifying } \\
\text { ointment } \\
\text { Glycerine/ } \\
\text { petroleum } \\
\text { Petroleum jelly }\end{array}$ & $\begin{array}{l}\text { Red Cross Children's } \\
\text { War Memorial } \\
\text { Hospital (South } \\
\text { Africa) }\end{array}$ & - & $\begin{array}{l}\text { Mild-moderate } \\
\qquad \mathrm{AD} \\
\text { Aged } 1-12 \text { years } \\
n=80\end{array}$ & UK Working Party \\
\hline $\begin{array}{l}\text { Kanehara et al. } \\
\text { [14] }\end{array}$ & $\begin{array}{l}\text { Borage oil (coated } \\
\text { on undershirts) }\end{array}$ & Unspecified & $\begin{array}{l}\text { Non-coated } \\
\text { placebo } \\
\text { undershirts }\end{array}$ & $\begin{array}{l}\text { Mild-moderate } \\
\quad \text { AD } \\
\text { Aged } 1-10 \text { years } \\
n=32\end{array}$ & Hanifin and Rajka \\
\hline
\end{tabular}


Table 1 continued

\begin{tabular}{|c|c|c|c|c|c|}
\hline References & Emollient(s) & $\begin{array}{l}\text { Study setting } \\
\text { (country) }\end{array}$ & $\begin{array}{l}\text { Non-emollient } \\
\text { comparator }\end{array}$ & $\begin{array}{l}\text { Patient } \\
\text { population }\end{array}$ & $\begin{array}{l}\text { Eczema diagnostic } \\
\text { criteria }\end{array}$ \\
\hline $\begin{array}{l}\text { Korting et al. } \\
\quad[15]\end{array}$ & $\begin{array}{l}\text { Verum (pale } \\
\text { sulfonated shale } \\
\text { oil cream } 4 \% \text { ) }\end{array}$ & $\begin{array}{l}\text { Outpatient centrers } \\
\text { (Germany) }\end{array}$ & "Vehicle" & $\begin{array}{l}\text { Mild-moderate } \\
\qquad \mathrm{AD} \\
\text { Aged } 0-12 \text { years } \\
n=99\end{array}$ & Unspecified \\
\hline $\begin{array}{l}\text { Korting et al. } \\
\text { [19] }\end{array}$ & $\begin{array}{c}\text { Hamamelis } \\
\text { distillate }\end{array}$ & Unspecified & $\begin{array}{l}\text { Hydrocortisone } \\
\text { cream on the } \\
\text { other side } \\
\text { "Drug-free vehicle" } \\
\text { (unspecified) }\end{array}$ & $\begin{array}{l}\text { Moderately } \\
\text { severe atopic } \\
\text { eczema } \\
\text { Aged } \\
18-62 \text { years } \\
n=72\end{array}$ & Hanifin and Rajka \\
\hline $\begin{array}{l}\text { Lodén et al. } \\
\text { [22] }\end{array}$ & $\begin{array}{l}\text { Glycerin cream } \\
\text { Urea cream }\end{array}$ & Unspecified & $\begin{array}{l}\text { "Placebo" } \\
\text { (unspecified) }\end{array}$ & $\begin{array}{l}\mathrm{AD} \\
\text { Adults } \\
n=197\end{array}$ & Unspecified \\
\hline $\begin{array}{l}\text { Lynde and } \\
\text { Andriessen } \\
{[29]}\end{array}$ & $\begin{array}{l}\text { CeraVe } \\
\text { Moisturizing } \\
\text { Cream }\end{array}$ & Unspecified & - & $\begin{array}{l}\text { Mild-moderate } \\
\text { AD } \\
\text { Fitzpatrick skin } \\
\text { types I-III } \\
\text { Children and } \\
\text { adults } \\
n=151\end{array}$ & Unspecified \\
\hline $\mathrm{Na}$ et al. [26] & Atobarrier Cream & Unspecified & - & $\begin{array}{l}\text { Mild-moderate } \\
\qquad \mathrm{AD} \\
\text { Aged } 5-19 \text { years } \\
n=30\end{array}$ & Unspecified \\
\hline $\begin{array}{l}\text { Ruzicka et al. } \\
\text { [20] }\end{array}$ & $\begin{array}{l}\text { Ointment base } \\
\text { containing } \\
\text { propylene } \\
\text { carbonate }\end{array}$ & Unspecified & $\begin{array}{c}0.03 \% \text { tacrolimus, } \\
0.1 \% \text { tacrolimus, } \\
0.3 \% \text { tacrolimus }\end{array}$ & $\begin{array}{l}\text { Moderate-severe } \\
\quad \mathrm{AD} \\
\text { Aged } \\
13-60 \text { years } \\
n=215\end{array}$ & $\begin{array}{l}\text { Rajka and } \\
\text { Langeland }\end{array}$ \\
\hline $\begin{array}{l}\text { Seghers et al. } \\
{[24]}\end{array}$ & $\begin{array}{l}\text { Curel Moisture } \\
\text { Cream }\end{array}$ & $\begin{array}{l}\text { National Skin Center } \\
\text { (Singapore) }\end{array}$ & - & $\begin{array}{l}\text { Stable, mild- } \\
\text { moderate AD } \\
\text { Aged } 7-60 \text { years } \\
n=40\end{array}$ & UK Working Party \\
\hline
\end{tabular}


Table 1 continued

\begin{tabular}{|c|c|c|c|c|c|}
\hline References & Emollient(s) & $\begin{array}{l}\text { Study setting } \\
\text { (country) }\end{array}$ & $\begin{array}{l}\text { Non-emollient } \\
\text { comparator }\end{array}$ & $\begin{array}{l}\text { Patient } \\
\text { population }\end{array}$ & $\begin{array}{l}\text { Eczema diagnostic } \\
\text { criteria }\end{array}$ \\
\hline $\begin{array}{l}\text { Shimelis et al. } \\
{[16]}\end{array}$ & $\begin{array}{l}\text { 10\% Chamomile } \\
\text { hydro-alcoholic } \\
\text { extract cream }\end{array}$ & $\begin{array}{l}\text { Mobile } \\
\text { dermatological } \\
\text { centers (Ethopia) }\end{array}$ & $\begin{array}{l}\text { "Placebo" } \\
\quad \text { (unspecified) }\end{array}$ & $\begin{array}{l}\text { AD } \\
\text { Aged } 1-78 \text { years } \\
n=11\end{array}$ & $\begin{array}{c}\text { Dermatologist } \\
\text { determined }\end{array}$ \\
\hline $\begin{array}{l}\text { Simpson et al. } \\
{[8]}\end{array}$ & $\begin{array}{l}\text { Cetaphil } \\
\text { Restoraderm }\end{array}$ & $\begin{array}{l}\text { Pro-DERM Institute } \\
\text { (Germany) }\end{array}$ & Untreated area & $\begin{array}{l}\text { “Controlled } \mathrm{AD} \text { ” } \\
\text { (no active } \\
\text { lesions in } \\
\text { target area) } \\
\text { Aged } \\
18-65 \text { years } \\
\mathrm{n}=20\end{array}$ & Unspecified \\
\hline $\begin{array}{l}\text { Stainer et al. } \\
\quad[17]\end{array}$ & $\begin{array}{l}\text { Altoderm lotion } \\
\text { ( } 4 \% \text { sodium } \\
\text { cromoglicate) }\end{array}$ & $\begin{array}{l}3 \text { NHS hospital } \\
\text { outpatient clinics, } 1 \\
\text { specialist clinical } \\
\text { research center } \\
\text { (England) }\end{array}$ & $\begin{array}{l}\text { "Placebo" (base } \\
\text { lotion alone) }\end{array}$ & $\begin{array}{l}\text { AD } \\
\text { Aged } 2-12 \text { years } \\
n=114\end{array}$ & UK Working Party \\
\hline Tan et al. [9] & $\begin{array}{l}\text { "Triclosan- } \\
\text { containing } \\
\text { emollient" }\end{array}$ & $\begin{array}{l}\text { National Skin Center } \\
\text { (Singapore) }\end{array}$ & "Vehicle" & $\begin{array}{l}\text { Mild-moderate } \\
\text { AD } \\
\text { Aged } \\
12-40 \text { years } \\
n=60\end{array}$ & Hanifin and Rajka \\
\hline $\begin{array}{l}\text { Udompatajkul } \\
\text { and } \\
\text { Srisatwaja } \\
{[18]}\end{array}$ & $\begin{array}{l}\text { Eucerin Soothing } \\
\text { Lotion } 12 \% \\
\text { omega } \\
\text { (Licochalcone) }\end{array}$ & Unspecified & $\begin{array}{l}\text { 1\% } \\
\text { Hydrocortisone } \\
\text { lotion }\end{array}$ & $\begin{array}{l}\text { Mild-moderate } \\
\qquad \mathrm{AD} \\
\text { Aged } 2-15 \text { years } \\
n=30\end{array}$ & Hanifin and Rajka \\
\hline
\end{tabular}

$A D$ Atopic dermatitis

studies did not specify how the diagnosis of eczema was confirmed $[7,8,10,13,15,22,23$, $25,26,29,30]$. Six (25\%) of the studies used Hanifin and Rajka criteria [9, 14, 18, 19, 21, 27] and four (17\%) used the UK Working Party's Group criteria [11, 12, 17, 24]. The eczema of participants in one (4\%) study was diagnosed by a dermatologist [16], in one (4\%) study diagnosis was based on Rajka and Langeland criteria [20] and in one (4\%) study diagnosis was based on criteria of the Japanese Dermatological Association [23].

\section{Emollient Type and Application}

Across all 24 studies included in the review, 29 named emollients were evaluated (see Table 2). The most common types of emollient were creams $(15,52 \%)[9,11,12,15,16,19,21-26$, 28, 29], lotions $(3,10 \%)[7,17,18]$ and 
Table 2 Studies according to emollient type and constituents

\begin{tabular}{|c|c|c|c|c|c|c|c|}
\hline \multirow{2}{*}{$\begin{array}{l}\text { Type of } \\
\text { emollient }\end{array}$} & \multirow{2}{*}{$\begin{array}{l}\text { Number of } \\
\text { emollients }\end{array}$} & \multirow[t]{2}{*}{ References } & \multicolumn{5}{|c|}{ Main constituents of emollient } \\
\hline & & & Urea & Ceramide & Glycerol & Herbal $^{\mathbf{a}}$ & Other ${ }^{b}$ \\
\hline \multirow[t]{3}{*}{ Lotion } & 3 & Bissonnette et al. [7] & $\bullet$ & & & & \\
\hline & & Stainer et al. [17] & & & & & $\bullet$ \\
\hline & & $\begin{array}{l}\text { Udompatajkul and Srisatwaja } \\
\text { [18] }\end{array}$ & & & & & $\bullet$ \\
\hline \multirow[t]{14}{*}{ Cream } & 15 & Chamlin et al. [28] & & $\bullet$ & & & \\
\hline & & Seghers et al. [24] & & $\bullet$ & & & \\
\hline & & Draelos and Raymond [25] & & $\bullet$ & & & \\
\hline & & Lynde and Andriessen [29] & & $\bullet$ & & & \\
\hline & & Boralevi et al. [11] & & & • & & \\
\hline & & Hashizume et al. [23] & & & 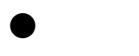 & & \\
\hline & & Hlela et al. [12] & & & ○ & & \\
\hline & & Lodén et al. [22] & $\bullet$ & & 0 & & \\
\hline & & Korting et al. [19] & & & & $\bullet$ & \\
\hline & & Shimelis et al. [16] & & & & $\bullet$ & \\
\hline & & Korting et al. [15] & & & & & $\bullet$ \\
\hline & & Boguniewicz et al. [21] & & & & & $\bullet$ \\
\hline & & $\mathrm{Na}$ et al. [26] & & & & & $\bullet$ \\
\hline & & Tan et al. [9] & & & & & $\bullet$ \\
\hline Gel & 1 & Draelos [27] & & & & & $\bullet$ \\
\hline \multirow[t]{4}{*}{ Ointment } & 4 & Haider [10] & & & & & $\bullet$ \\
\hline & & Chishti et al. [30] & & & & $\bullet$ & \\
\hline & & Hlela et al. [12] & & & & & $\bullet$ \\
\hline & & Ruzicka et al. [20] & & & & & $\bullet$ \\
\hline Jelly & 1 & Hlela et al. [12] & & & & & ○ \\
\hline $\begin{array}{l}\text { Coated } \\
\text { undershirt }\end{array}$ & 1 & Kanehara et al. [14] & & & & $\bullet$ & \\
\hline \multirow[t]{4}{*}{ Unknown } & 4 & Bissonnette et al. [7] & 0 & & & & \\
\hline & & Simpson et al. [8] & & $\bullet$ & & & \\
\hline & & Hlela et al. [12] & & & & & - \\
\hline & & Gandy et al. [13] & & & & & $\bullet$ \\
\hline
\end{tabular}


Table 2 continued

\begin{tabular}{|c|c|c|c|c|c|c|c|}
\hline \multirow{2}{*}{$\begin{array}{l}\text { Type of } \\
\text { emollient }\end{array}$} & \multirow{2}{*}{$\begin{array}{l}\text { Number of } \\
\text { emollients }\end{array}$} & \multirow[t]{2}{*}{ References } & \multicolumn{5}{|c|}{ Main constituents of emollient } \\
\hline & & & $\overline{\text { Urea }}$ & Ceramide & Glycerol & Herbal $^{\mathbf{a}}$ & Other $^{\mathbf{b}}$ \\
\hline Total & 29 & & 3 & 5 & 4 & 4 & 13 \\
\hline
\end{tabular}

a Herbal: Pale sulfonated shale oil cream 4\% [15]; 10\% chamomile hydro-alcoholic extract cream [16]; hamamelis distillate [19]; Dermovix [30]

b Other: “Triclosan-containing emollient" [9]; CHD-FA 3.5\% (carbohydrate-derived fulvic acid) [13]; borage oil [14]; 12\% omega (Licochalcone A Lotion) [18]; propylene carbonate [20]; 10\% sodium cromoglycate ointment [10]; Altoderm lotion (4\% sodium cromoglicate) [17]

ointments $(4,14 \%)[10,12,30,20]$. In one study the emollient was a gel [27], in one study it was a "jelly" [12] and one study explored emollients applied to a coated undershirt [14]. The formulation of four (14\%) emollients could not be determined [7, 8, 12, 13].

When grouping the emollients according to their provenance or humectants (Table 2), three $(10 \%)$ contained urea $[7,22]$, five $(17 \%)$ contained ceramide $[8,24,25,28,29]$, four $(14 \%)$ contained glycerol $[11,12,22,23]$ and four $(14 \%)$ had a herbal basis $[14,16,25,30]$. Thirteen (45\%) were classified as "other" $[9,10,12,13,15,17,18,20,21,26,27]$.

\section{Adverse Events Associated With Emollients According to Constituents}

No serious treatment-related adverse events were reported for any emollient. The proportion of participants experiencing treatment-related adverse events varied between 2.4 and $58.8 \%$ $[9,11,12,15,17,20,24,26,27]$. The most common adverse events were skin related and often mild. The range of participants experiencing non-treatment-related adverse events varied between 4.2 and $43.1 \%$ [11, 15, 17, 19, 20] (see Table 3).

\section{Urea-Containing Emollients}

Participants using Iso-Urea or $10 \%$ urea lotion [7] reported 21 adverse events not related to the study treatment, including common colds and headaches (Table A1, ESM Appendix 2). The five treatment-related adverse events reported were all related to the skin. There was one withdrawal due to adverse events with participants using Iso-Urea (erythema on eczema plaques) and two withdrawals due to adverse events with participants using 10\% urea lotion (irritant contact dermatitis and pruritus). A study into urea cream [22] only reported on adverse skin reactions, which included smarting, stinging, itching and dryness/irritation. No serious adverse events were reported for any of the urea-containing emollients.

\section{Ceramide-Containing Emollients}

Of the five ceramide-containing emollients evaluated, four (Cetaphil Restoraderm Body Moisturizer [8], NeoCera [25], CeraVe Moisturizing Cream [29], TriCeram Cream [28]) were not associated with any no treatment-related adverse events (Table A2, ESM Appendix 2). Seven participants using Curel Moisture Cream [28] experienced pruritus and one experienced a warm sensation after application. There was one withdrawal due to adverse events from the use of Curel Moisture Cream (worsening of rashes and acneiform papules on the face). No serious adverse events were reported for any of the ceramide-containing emollients.

\section{Glycerol-Containing Emollients}

Of the four glycerol-containing emollients evaluated, treatment-related adverse events were only not reported by participants using $\mathrm{N}$-acetylhydroxyproline cream (AHYP) [23] (Table A3, ESM Appendix 2). Three participants experienced treatment-related adverse events using Dexeryl [11] (mild to moderate erythema, burning, pruritus); one participant using glycerine/ 


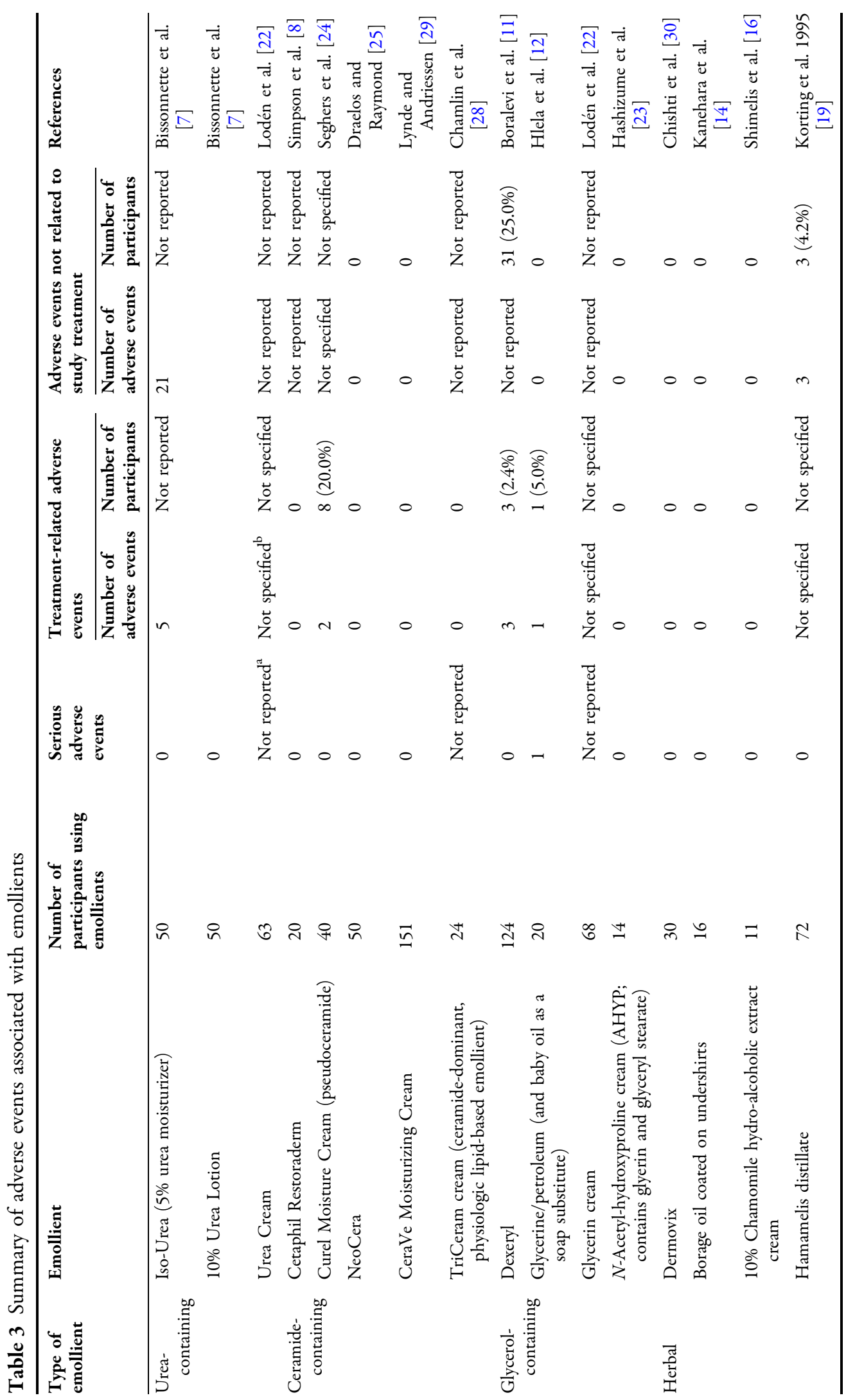




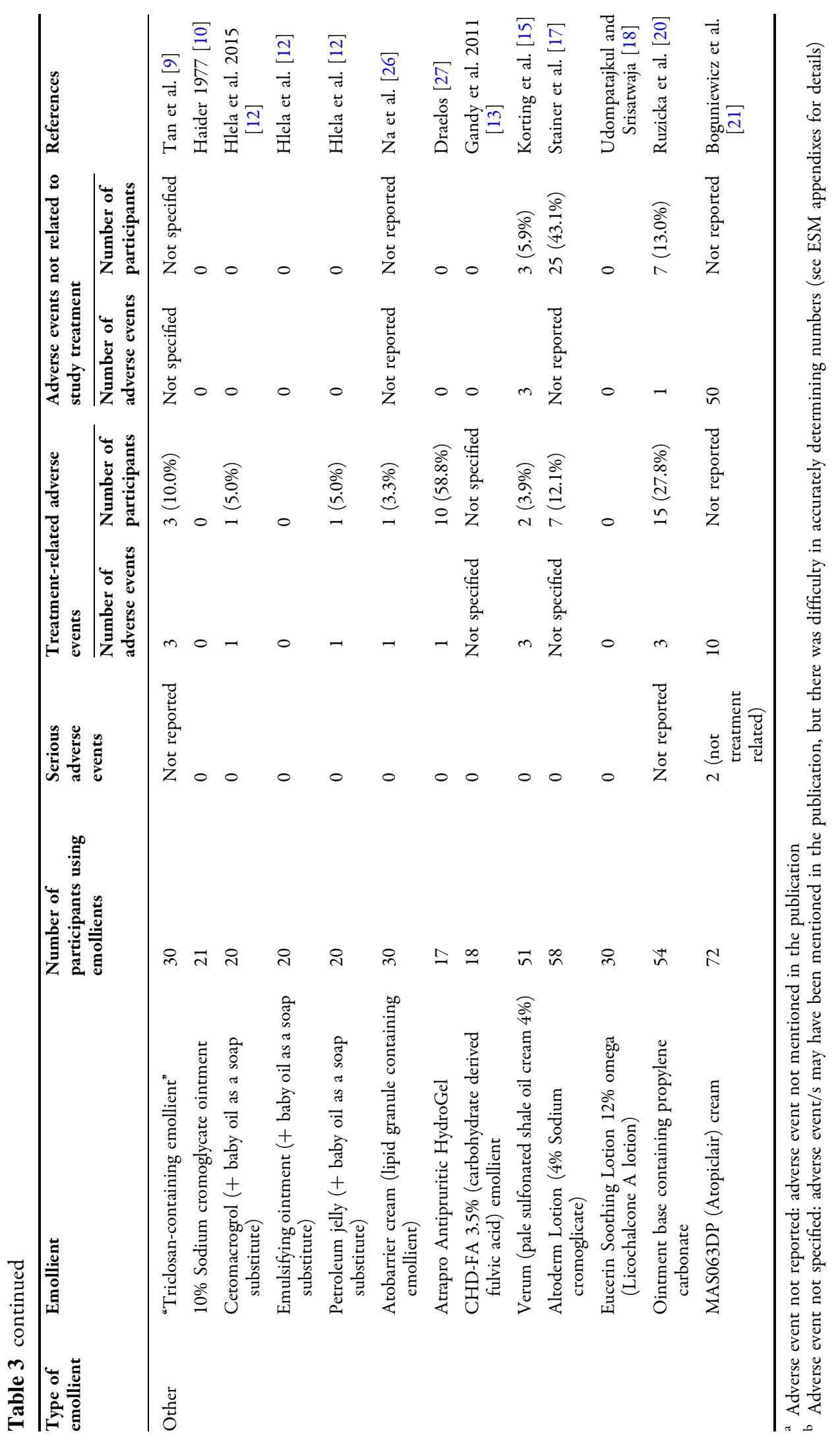


petroleum (and baby oil as a soap substitute) [12] experienced itching, although this was perceived to be with associated with the baby oil. Participants using glycerin cream [22] only reported on adverse skin reactions, which included smarting, stinging, itching and dryness/irritation. Thirtyone participants experienced non-treatment-related adverse events when using Dexeryl [11]. No serious adverse events were reported for any of the glycerol-containing emollients.

\section{Herbal Emollients}

Treatment-related or non-treatment-related adverse events were not reported for three of the four herbal emollients evaluated: Dermovix [30], borage oil coated on undershirts [14] and $10 \%$ chamomile hydro-alcoholic extract cream [16] (Table A4, ESM Appendix 2). In a study on Hamamelis distillate [19], five participants reported experiencing itching, erythema, stinging, lichenification or dry skin. Across the two treatment groups, there were three non-treatment-related adverse events (herpes simplex infection, cystitis and bronchitis). No serious adverse events were reported for any of the herbal emollients.

\section{Other Emollients}

Three of the 13 emollients classified as "other" (10\% sodium cromoglycate ointment [10], emulsifying ointment [12], Eucerin Soothing Lotion $12 \%$ omega [18]) did not result in any treatment-related adverse events (Table A5, ESM Appendix 2). The range of treatment-related adverse events reported was from 5.0 to $58.8 \%$. Three participants experienced transient pain after the application of a triclosan-containing emollient [9]. One participant using Cetomacrogrol (and baby oil as a soap substitute) [12] and one participant using petroleum jelly (and baby oil as a soap substitute) [12] experienced itching; however this was perceived to be associated with the baby oil. One participant withdrew due to a treatment-related adverse event (erythema after use) when using Atobarrier Cream [26]. Ten participants experienced mild post application skin dryness after the use of Atrapro Antipruritic HydroGel [27]. The only treatment-related adverse events reported after use of CHD-FA 3.5\% (carbohydrate-derived fulvic acid) emollient was a short-lived burning sensation [13]. The use of Verum [15] resulted in two withdrawals due to adverse events (itch, erythema and spreading of the eczema). For Altoderm lotion [17] there were seven reports of treatment-related adverse events (erythema and pruritus and application site burning) and one report of an allergic reaction (stinging, itching, redness, skin evolved into eczema). Fifteen participants reported treatment-related adverse events (sensation of burning at the site of application, pruritus and erythema) when using an ointment base containing propylene carbonate [20]. Ten adverse events were reported by participants who had used MAS063DP (Atopiclair) Cream [21].

Participants using seven of the emollients (10\% sodium cromoglycate ointment [10], Cetomacrogrol [12], Emulsifying ointment [12], petroleum jelly [12], Atrapro Antipruritic HydroGel [27], CHD-FA 3.5\% [13], Eucerin Soothing Lotion $12 \%$ omega [18]) did not have any non-treatment-related adverse events. The range of non-treatment-related adverse events was between 6 and 43\%. Three participants using Verum [15] reported non-treatment-related adverse events (bronchitis, worsened teething and concussion), and 22 participants using Altoderm Lotion reported non-treatmentrelated adverse events [17]. Seven participants reported a non-treatment-related adverse event (exacerbation of atopic dermatitis) when using an ointment base containing propylene carbonate [20]. Fifty non-treatment-related adverse events were reported by participants who used MAS063DP (Atopiclair) Cream [21].

No serious treatment-related adverse events were recorded in the "other" emollients. Boguniewicz et al. [21] reported two serious (as determined by the author) non-treatment-related adverse events (tonsillectomy and acute asthma exacerbation); however, it was not possible to determine whether these occurred in the treatment group (MAS063DP [Atopiclair] Cream) or the vehicle group. 


\section{DISCUSSION}

\section{Summary of Main Findings}

Across the 24 studies identified, 29 emollients were evaluated. Most of these 24 studies were RCTs, and there was a mix of studies involving children, adults or both. The location of many studies was unspecified, but the most common setting was specialist care. The criteria for diagnosing eczema was also not stated in many of the papers. The most common type of emollient was cream, with the formulation of some not described.

No serious treatment-related adverse events were reported for any of emollients. The proportion of participants experiencing treatmentrelated adverse events varied between 2.4 and $58.8 \%[9,11,12,15,17,20,24,26,27]$, and the most common adverse events were skin related and often mild. The range of participants experiencing non-treatment-related adverse events varied between 4.2 and $43.1 \%$ [11, 15, 17, 19, 20] .

\section{Strengths and Limitations}

We believe this our review is the first review with the specific aim to quantify and compare the frequency of adverse events between different emollients across all types of published studies. We followed a "restricted review" approach [6] and were able to rapidly identify and summarize relevant international publications involving all types of studies. However, due to resource limitations we did not attempt to apply any criteria to assess the quality of the research that we identified. We found that few studies reported on adverse events and of those that did, data was generally of poor quality. It was difficult to interpret results and compare emollients due to missing data, confusion over whether adverse events referred to the treatment or control and inconsistency in reporting by subject or total numbers of the adverse event. Several studies compared the "active" emollient to a "vehicle," but the extent to which such a comparator is "inert" is arguable. We adopted the descriptions of the emollients given by the authors of the included papers at face value and summarized these accordingly, such as, for example, "urea containing," "herbal". As a result, it is possible that some emollients may have been "misclassified"; however, reporting of the original studies means we were unable to better delineate similarities and differences between the emollients.

Limitations of this review itself are the searching of only one database, exclusion of papers not written in English and inability to retrieve the full text of some articles which potentially could have been included. Restrictions to the search criteria ("eczema" and "adverse events") will have excluded a number of potentially relevant studies in which emollients were evaluated in people without eczema and in those where data on adverse events are reported in the manuscript but not the title or abstract. This limitation has been confirmed by the omission of some papers from the results of the search strategy which were included in the recent Cochrane Review [1]. Only one reviewer screened all the titles/abstracts and retrieved papers, but in cases of uncertainty, eligibility for inclusion was discussed with another author.

The wide variation observed in the proportion of participants experiencing an adverse event across the included studies may reflect a true difference between the different emollients, the population studied, how different researchers collected adverse event data and/or the number of participants in the study. In other words, we have presented adverse events as proportions of the total number of participants in each study, but the smaller the denominator, the greater the potential error around that estimate.

\section{Comparison with Existing Literature}

In common with previous systematic reviews that have included emollients for eczema, we found that adverse reporting in studies of emollients is poor and when reporting is present, the data suggest that in general these events are of low frequency and mild [1, 31]. Van Zuuren et al. [1] reported that the relative risks of an adverse event from all emollients was 24 per 100 (95\% confidence interval 19-30) 
participants compared with 23 per 100 with vehicle, placebo or no moisturizer (10 RCTs, 1275 participants, follow-up range from 4 weeks to 6 months) but that there were more adverse events associated with urea-containing creams (65 per 100 participants in 1 RCT in which 129 participants were compared with placebo) and oat-containing moisturizers ( 9 per 100 participants in 1 RCT in which 173 participants were compared with no moisturizer).

\section{CONCLUSIONS}

Due to the limitations of both this review and the available literature, our findings should be interpreted with caution. Nevertheless, they provide a basis for further detailed research, with recommendations for a full systematic review of adverse effects associated with emollient use and better design and reporting of future studies that evaluate emollients, including adverse events.

Based on the findings of this restricted review, clinicians and patients can be reassured that the emollients studied appear to be generally safe to use. However, the emollients described in these studies are not in common use in many countries. Until further data become available, patients and clinicians should be encouraged to report adverse events via their national systems, such as the Yellow Card scheme [32].

\section{ACKNOWLEDGEMENTS}

Funding. $\mathrm{AB}$ was funded by an NIHR School for Primary Care Research (SPCR) internship. MJR is funded by an NIHR Post-Doctoral Research Fellowship (PDF-2014-07-013). The views expressed in this publication are those of the authors and not necessarily those of the NHS, the National Institute for Health Research or the Department of Health and Social Care. No funding or sponsorship was received for the publication of this article.
Authorship. All named authors meet the International Committee of Medical Journal Editors (ICMJE) criteria for authorship for this article, take responsibility for the integrity of the work as a whole, and have given their approval for this version to be published.

Disclosures. Matthew Ridd is chief investigator on the BEE study (funded by NIHR Health Technology Assessment, ref 15/130/07, www. bristol.ac.uk/bee-study) which is comparing the effectiveness and acceptability of four different types of emollient for children with eczema. Alisha Bhanot and Alyson Huntley have nothing to disclose.

Compliance with Ethics Guidelines. This article is based on previously conducted studies and does not contain any studies with human participants or animals performed by any of the authors, so ethical approval was not required.

Data Availability. All data are submitted as supplementary files.

Open Access. This article is distributed under the terms of the Creative Commons Attribution-NonCommercial 4.0 International License (http://creativecommons.org/licenses/ by-nc/4.0/), which permits any noncommercial use, distribution, and reproduction in any medium, provided you give appropriate credit to the original author(s) and the source, provide a link to the Creative Commons license, and indicate if changes were made.

\section{REFERENCES}

1. van Zuuren EJ, Fedorowicz Z, Christensen R, Lavrijsen APM, Arents BWM. Emollients and moisturisers for eczema. Cochrane Database of Syst Rev. 2017:CD012119 https://doi.org/10.1002/14651858. cd012119.pub2.

2. Eichenfield LF, Tom WL, Berger TG, et al. Guidelines of care for the management of atopic dermatitis: part 2: management and treatment of atopic dermatitis with topical therapies. J Am Acad Dermatol. 2014;71(1):116-32. 
3. Joint Formulary Committee. British National Formulary: emollient and barrier preparations. Available at: https://bnf.nice.org.uk/treatmentsummary/emollient-and-barrier-preparations.html. Accessed 3 Dec 2018.

4. Hengge U, Ruzicka T, Schwartz R, Cork M. Adverse effects of topical glucocorticosteroids. J Am Acad Dermatol. 2006;54(1):1-15.

5. Cury Martins J, Martins C, Aoki V, Gois AFT, Ishii HA, da Silva EMK. Topical tacrolimus for atopic dermatitis. Cochrane Database of Syst Rev. 2015:CD009864. 14651858.cd009864.pub2. https://doi.org/10.1002/

6. Plüddemann A, Aronson J, Onakpoya I, Heneghan C, Mahtani K. Redefining rapid reviews: a flexible framework for restricted systematic reviews. BMJ Evid Based Med. 2018;23(6):201-3. https://doi.org/ 10.1136/bmjebm-2018-110990.

7. Bissonnette R, Maari C, Provost N, et al. A doubleblind study of tolerance and efficacy of a new ureacontaining moisturizer in patients with atopic dermatitis. J Cosmet Dermatol. 2010;9:16-21.

8. Simpson E, Bohling A, Bielfeldt S, Bosc C, Kerrouche $\mathrm{N}$. Improvement of skin barrier function in atopic dermatitis patients with a new moisturizer containing a ceramide precursor. J Dermatol Treat. 2013;24:122-5.

9. Tan W, Suresh S, Tey H, Chiam L, Goon A. A randomized double-blind controlled trial to compare a triclosan-containing emollient with vehicle for the treatment of atopic dermatitis. Clin Exp Dermatol. 2010;35:e109-12.

10. Haider S. Treatment of atopic eczema in children: clinical trial of $10 \%$ sodium cromoglycate ointment. BMJ. 1977;1:1570-2.

11. Boralevi F, Saint Aroman M, Delarue A, et al. Longterm emollient therapy improves xerosis in children with atopic dermatitis. J Eur Acad Dermatol Venereol. 2014;28:1456-62.

12. Hlela C, Lunjani N, Gumedze F, Kakande B, Khumalo N. Affordable moisturisers are effective in atopic eczema: a randomised controlled trial. S Afr Med J. 2015;2015(105):780-4.

13. Gandy J, Snyman J, Van Rensburg C. Randomized, parallel-group, double-blind, controlled study to evaluate the efficacy and safety of carbohydratederived fulvic acid in topical treatment of eczema. Clin Cosmet Investig Dermatol. 2011;4:145-8.

14. Kanehara S, Ohtani T, Uede K, Furukawa F. Clinical effects of undershirts coated with borage oil on children with atopic dermatitis: a double-blind, placebo-controlled clinical trial. J Dermatol. 2007;34:811-5.

15. Korting H, Schollmann C, Cholcha W, Wolff L. Efficacy and tolerability of pale sulfonated shale oil cream $4 \%$ in the treatment of mild to moderate atopic eczema in children: a multicentre, randomized vehicle-controlled trial. J Eur Acad Dermatol Venereol. 2010;24:1176-82.

16. Shimelis N, Asticcioli S, Baraldo M, Tirillini B, Lulekal E, Murgia V. Researching accessible and affordable treatment for common dermatological problems in developing countries. An Ethiopian experience. Int J Dermatol. 2012;51:790-5.

17. Stainer R, Matthews S, Arshad S, et al. Efficacy and acceptability of a new topical skin lotion of sodium cromoglicate (Altoderm) in atopic dermatitis in children aged 2-12 years: a double-blind, randomized, placebo-controlled trial. $\mathrm{Br} \mathrm{J}$ Dermatol. 2005;152:334-41.

18. Udompataikul M, Srisatwaja W. Comparative trial of moisturizer containing licochalcone A vs. hydrocortisone lotion in the treatment of childhood atopic dermatitis: a pilot study. J Eur Acad Dermatol Venereol. 2011;25:660-5.

19. Korting H, Schafer-Korting M, Klovekorn W, Klovekorn G, Martin C, Laux P. Comparative efficacy of hamamelis distillate and hydrocortisone cream in atopic eczema. Eur J Clin Pharmacol. 1995;48:461-5.

20. Ruzicka T, Bieber T, Schopf E, et al. A short-term trial of tacrolimus ointment for atopic dermatitis. European Tacrolimus Multicenter Atopic Dermatitis Study Group. N Engl J Med. 1997;337:816-21.

21. Boguniewicz M, Zeichner J, Eichenfield L, et al. MAS063DP is effective monotherapy for mild to moderate atopic dermatitis in infants and children: a multicenter, randomized, vehicle-controlled study. J Pediatr. 2008;2008(152):854-9.

22. Lodén M, Andersson A, Anderson C, et al. A doubleblind study comparing the effect of glycerin and urea on dry, eczematous skin in atopic patients. Acta Dermato-Venereol. 2002;82(1):45-7.

23. Hashizume E, Nakano T, Kamimura A, Morishita K. Topical effects of $\mathrm{N}$-acetyl-L-hydroxyproline on ceramide synthesis and alleviation of pruritus. Clin Cosmet Investig Dermatol. 2013;6:43-9.

24. Seghers A, Cai S, Ho M, et al. Evaluation of a pseudoceramide moisturizer in patients with mildto-moderate atopic dermatitis. Dermatol Ther. 2014;4:83-92. 
25. Draelos Z, Raymond I. The efficacy of a ceramidebased cream in mild-to-moderate atopic dermatitis. J Clin Aesth Dermatol. 2018;11:30-2.

26. Na J, Hwang J, Park H, et al. A new moisturizer containing physiologic lipid granules alleviates atopic dermatitis. J Dermatol Treat. 2010;21:23-7.

27. Draelos Z. Antipruritic hydrogel for the treatment of atopic dermatitis: an open-label pilot study. Cutis. 2012;2012(90):97-102.

28. Chamlin S, Kao J, Frieden I, et al. Ceramide-dominant barrier repair lipids alleviate childhood atopic dermatitis: changes in barrier function provide a sensitive indicator of disease activity. J Am Acad Dermatol. 2002;47(2):198-208.

29. Lynde C, Andriessen A. A cohort study on a ceramide-containing cleanser and moisturizer used for atopic dermatitis. Cutis. 2014;93:207-13.
30. Chishti M, Mohi-Ud-Din E, Usmanghani K, Nawaz A, Nazar H, Ahmad I. Comparative clinical efficacy and safety of coded herbal medicine Dermovix in the management of patients with atopic dermatitis versus allopathic medicine. Pak J Pharm Sci. 2015;2015(28):1655-63.

31. Nankervis H, Thomas $\mathrm{K}$, Delamere F, Barbarot S, Rogers N, Williams H. Scoping systematic review of treatments for eczema. Progr Grants Appl Res. 2016;4(7).

32. Medicines and Healthcare products Regulatory Agency (MHRA). Yellow card scheme. 2018. https:// yellowcard.mhra.gov.uk/. Accessed 4 Dec 2018. 